\title{
Discovering Partonic Rescattering in Light Nucleus Collisions
}

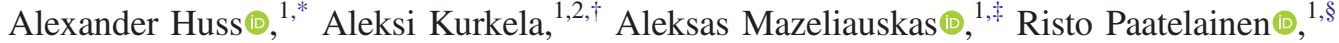 \\ Wilke van der Schee, ${ }^{1, \|}$ and Urs Achim Wiedemann ${ }^{1,}{ }^{1, \|}$ \\ ${ }^{1}$ Theoretical Physics Department, CERN, CH-1211 Genève 23, Switzerland \\ ${ }^{2}$ Faculty of Science and Technology, University of Stavanger, 4036 Stavanger, Norway
}

(Received 12 August 2020; accepted 30 March 2021; published 11 May 2021)

\begin{abstract}
We demonstrate that oxygen-oxygen collisions at the LHC provide unprecedented sensitivity to parton energy loss in a system whose size is comparable to those created in very peripheral heavy-ion collisions. With leading and next-to-leading order calculations of nuclear modification factors, we show that the baseline in the absence of partonic rescattering is known with up to $2 \%$ theoretical accuracy in inclusive oxygen-oxygen collisions. Surprisingly, a Z-boson normalized nuclear modification factor does not lead to higher theoretical accuracy within current uncertainties of nuclear parton distribution functions. We study a broad range of parton energy loss models and we find that the expected signal of partonic rescattering can be disentangled from the baseline by measuring charged hadron spectra in the range $20 \mathrm{GeV}<p_{T}<100 \mathrm{GeV}$.
\end{abstract}

DOI: 10.1103/PhysRevLett.126.192301

Introduction.-Evidence for the formation of deconfined QCD matter-the quark-gluon plasma-in nucleusnucleus (AA) collisions at the LHC and at the Relativistic Heavy Ion Collider comes from several classes of experimental signatures: the suppression of high-momentum hadronic yields ("parton energy loss"), the momentum anisotropy seen in multiparticle correlations ("collective flow"), the increased fraction of strange hadron yields ("strangeness enhancement"), the exponential spectra of electromagnetic probes ("thermal radiation"), and others [1-10]. Several of these findings signal the presence of partonic rescattering in the QCD medium produced in AA collisions. Even in smaller collision systems, in which interactions may be so feeble that the systems evolve close to free streaming, a smaller but nonvanishing strength of these signatures is expected.

Much experimental effort at the LHC has gone recently into characterizing emergent QCD medium properties as a function of the size of the collision system. Strangeness enhancement and collective flow have been observed in the most peripheral AA collisions, as well as in proton-nucleus $(p \mathrm{~A})$ and in proton-proton $(p p)$ collisions [11-14]. In marked contrast, no sign of parton energy loss has been observed within current measurement uncertainties in $p \mathrm{~A}$ collisions, and measurements in peripheral AA remain inconclusive because of large systematic uncertainties

Published by the American Physical Society under the terms of the Creative Commons Attribution 4.0 International license. Further distribution of this work must maintain attribution to the author(s) and the published article's title, journal citation, and DOI. Funded by SCOAP. (see Fig. 1). However, all parton energy loss models predict some (possibly small) signal in small collision systems. The experimental testing of this robust prediction is arguably one of the most important challenges of the future experimental heavy-ion programs $[15,16]$.

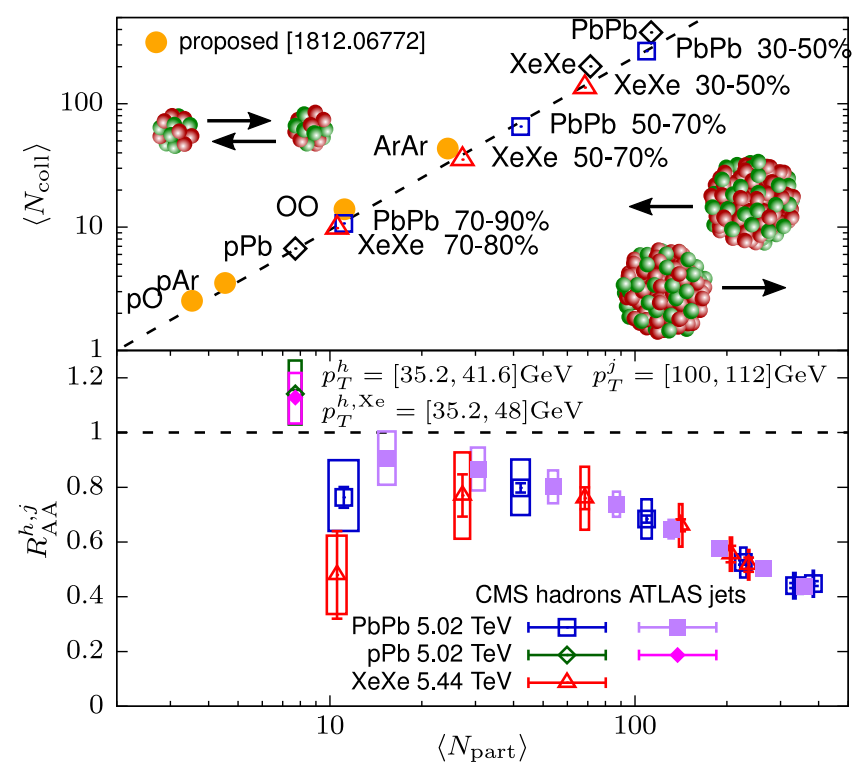

FIG. 1. (top) The number of binary collisions as a function of participant nucleons in minimum bias nucleus-nucleus, protonnucleus, and centrality selected heavy-ion collisions. (bottom) Measured hadron and jet nuclear modification factors in $\mathrm{PbPb}$, $\mathrm{XeXe}$, and $\mathrm{pPb}$ collisions [17-20]. Error bars are statistical, while boxes are the combined systematic, luminosity, and $\left\langle T_{\mathrm{AA}}\right\rangle$ uncertainties. $\left\langle T_{\mathrm{AA}}\right\rangle$ uncertainty dominates in peripheral AA collisions. 
In this Letter, we show how oxygen-oxygen (OO) collisions at the LHC provide a unique opportunity to discover (small) medium induced energy loss in small systems.

Nuclear modification factor.-The main signal for parton energy loss is the observed suppression of energetic particles in AA collisions. It is typically quantified by the nuclear modification factor

$$
R_{\mathrm{AA}}^{h, j}\left(p_{T}, y\right)=\frac{1}{\left\langle T_{\mathrm{AA}}\right\rangle} \frac{\left(1 / N_{\mathrm{ev}}\right) d N_{\mathrm{AA}}^{h, j} / d p_{T} d y}{d \sigma_{p p}^{h, j} / d p_{T} d y},
$$

which compares the differential yield in AA collisions to the yield in an equivalent number $\left\langle N_{\text {coll }}\right\rangle=\sigma_{p p}^{\text {inel }}\left\langle T_{\mathrm{AA}}\right\rangle$ of $p p$ collisions. Here, $\sigma_{p p}^{\text {inel }}$ is the total inelastic $p p$ cross section, $\left\langle T_{\mathrm{AA}}\right\rangle$ is the nuclear overlap function within a given centrality interval, and $N_{\mathrm{ev}}$ is the number of collision events in this centrality interval. $d N_{\mathrm{AA}}^{h, j} / d p_{T} d y$ is the differential yield of charged hadrons $(h)$ or calorimetrically defined jets $(j)$ produced in AA collisions at transverse momentum $p_{T}$ and longitudinal rapidity $y$, and $d \sigma_{p p}^{h, j} / d p_{T} d y$ is the corresponding differential $p p$ cross section.

The system size dependence of parton energy loss is typically studied in terms of the centrality dependence of $R_{\mathrm{AA}}\left(p_{T}, y\right)$. Experimentally, centrality is defined as the selected percentage of the highest multiplicity events of the total inelastic AA cross section. Theoretically, it is related by Glauber-type models to $\left\langle T_{\mathrm{AA}}\right\rangle$, to the mean number of participating nucleons $\left\langle N_{\text {part }}\right\rangle$, and to the mean number of nucleon-nucleon collisions $\left\langle N_{\text {coll }}\right\rangle$ [21-24]. As seen from the top panel of Fig. 1, inclusive (i.e., centrality averaged) $\mathrm{OO}$ collisions probe the system size corresponding to highly peripheral lead-lead $(\mathrm{PbPb})$ and xenon-xenon (XeXe) collisions.

The differential cross section $d \sigma_{p p}^{h, j}$ entering Eq. (1) can be measured precisely, and it can be calculated at sufficiently high $p_{T}$ with controlled accuracy in QCD perturbation theory. However, the nuclear overlap function $\left\langle T_{\mathrm{AA}}\right\rangle$ depends on the soft physics of total inelastic $p p$ cross sections and on the model dependent estimation of binary nucleon-nucleon collisions. Estimates of the uncertainties associated to $\left\langle T_{\mathrm{AA}}\right\rangle$ range from $3 \%$ in central to $15 \%$ in the peripheral $\mathrm{PbPb}$ collisions [17]. In addition, there are known event selection and geometry biases that in peripheral AA collisions complicate the model comparison of nuclear modification factors [24]. In this way, the characterization of a high-momentum transfer process becomes dependent on the modeling of low-energy physics whose uncertainties are difficult to estimate and to improve. This limits the use of Eq. (1) for characterizing numerically small medium modifications in very peripheral heavy-ion and $p \mathrm{~A}$ collisions. A centrality averaged measurement of Eq. (1) in OO collisions would have a smaller $\left\langle T_{\mathrm{AA}}\right\rangle$ uncertainty than $15 \%$, but soft physics assumptions remain [25].

It is of interest to characterize parton energy loss in the range of $\left\langle N_{\text {part }}\right\rangle \sim 10$ with measurements independent of soft physics assumptions. The study of inclusive, minimum bias $R_{\mathrm{AA}}^{h, j}$ in collisions of light nuclei allows for this since

$$
R_{\mathrm{AA}, \min \text { bias }}^{h, j}\left(p_{T}, y\right)=\frac{1}{A^{2}} \frac{d \sigma_{\mathrm{AA}}^{h, j} / d p_{T} d y}{d \sigma_{p p}^{h, j} / d p_{T} d y}
$$

is independent of $\left\langle T_{\mathrm{AA}}\right\rangle$. The system size is controlled by selecting nuclei with different nucleon number $A$. Proposed light-ion collisions with oxygen $A=16$ and $\operatorname{argon} A=40$ at the LHC provide a system size scan in the physically interesting region (see Fig. 1).

Perturbative benchmark calculations.-The ability to discover a small signal of high- $p_{T}$ partonic rescattering via Eq. (2) is now free from soft physics assumptions. It depends solely on the experimental precision of the measurement and on the accuracy with which theory can calculate the null hypothesis, i.e., the value of $R_{\mathrm{AA} \text {, min bias }}^{h, j}$ in the absence of partonic rescattering. This null hypothesis depends only on high-momentum transfer processes that can be computed with systematically improvable accuracy in collinearly factorized perturbative QCD. To determine the null hypothesis, we calculate inclusive jet cross section in $p p$ and $\mathrm{OO}$ collisions at $\sqrt{s_{N N}}=7 \mathrm{TeV}$ as the convolution of incoming parton distribution functions (PDFs) with hard matrix elements with the NNLOJET framework $[26,27]$ and using APPLfast interpolation tables [28]. For $p p$ collisions, cross section calculations provide quantitatively reliable predictions at next-to-leading order (NLO) and have been pushed to next-to-next-to-leading order accuracy or even beyond for many important processes. For nuclei, the nuclear modifications of the PDFs (nPDFs) are currently available up to NLO accuracy, so we restrict calculations of Eq. (2) up to this order.

Results for the minimum bias nuclear modification factor of jets are shown in Fig. 2. The uncertainties in the proton $\mathrm{PDF}$ and in the fixed-order perturbative calculation were estimated using the free proton PDF sets provided by CT14 [29] and by independently varying the factorization and renormalization scales by factors $\frac{1}{2}$ and 2 while imposing $\frac{1}{2} \leq \mu_{R} / \mu_{F} \leq 2$. For leading order (LO) and NLO calculations, these theoretical uncertainties enter the numerator and denominator of Eq. (2) and are found to cancel to a large extent in the ratio. We checked that parton shower (PS) and hadronization effects also largely cancel using the NLO+PS implementation of POWHEG+PYTHIA8 [30].

Uncertainties of nuclear modification of the free proton PDFs, however, enter only in the numerator of Eq. (2). They were calculated using nPDF sets from EPPS16 global fit, including a subset of LHC data on electroweak boson and dijet production in $p \mathrm{~Pb}$ [31]. nPDFs constitute the largest theoretical uncertainty, increasing from $\sim 2 \%$ at 


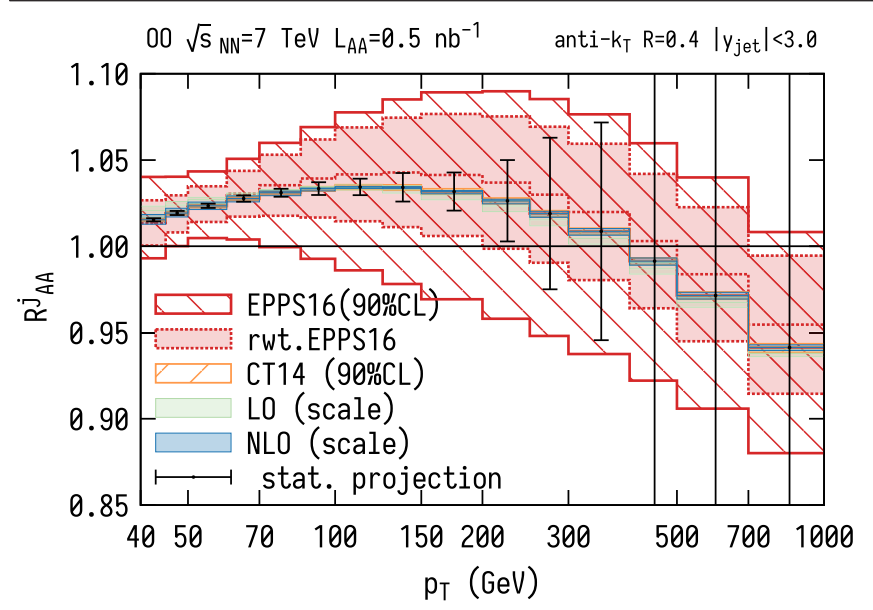

FIG. 2. Minimum bias jet nuclear modification factor Eq. (2) for $\mathrm{OO}$ collisions in the absence of parton rescattering. The red bands show nPDF $90 \%$ confidence level (CL) (reweighting is done by including additional $p \mathrm{~Pb}$ dijet data). Proton PDF (orange) and scale (green and blue) uncertainties are fully correlated and cancel. Error bars illustrate statistical uncertainties for OO mock data at $100 \%$ efficiency (see text for other uncertainties).

$p_{T}=50 \mathrm{GeV}$ to $\sim 7 \%$ for $p_{T}>200 \mathrm{GeV}$. Compared to a conservative $15 \%$ uncertainty estimate on the modeling of $\left\langle T_{\mathrm{AA}}\right\rangle$ for very peripheral heavy-ion collisions, they are approximately 4 times smaller for $p_{T}<100 \mathrm{GeV}$. Moreover, nPDF uncertainties can be reduced by including additional LHC data. We show this by reweighting nPDF uncertainties with Compact Muon Solenoid Experiment dijet data [32] (following the work of Ref. [33,34]; see the Supplemental Material [35]). The nPDF 90\% confidence level band in Fig. 2 then shrinks to 1\% (4\%) at low (high) $p_{T}$, respectively. This demonstrates that the null hypothesis in the absence of parton energy loss is known with much higher accuracy from Eq. (2) than from the centrality dependent measurements of Eq. (1).

To gain insight into whether this higher theoretical accuracy can be exploited in an upcoming OO run, we have overlaid in Fig. 2 statistical uncertainties of OO mock data for an integrated luminosity of $\mathcal{L}_{\mathrm{AA}}=0.5 \mathrm{nb}^{-1}$ corresponding to a few hours of stable beam in the "moderately optimistic" running scenario of Ref. [15]. The errors displayed on the mock data do not account for several sources of experimental uncertainties that can only be determined with detailed knowledge of the detectors and the machine. There are indications that the systematic experimental uncertainties entering Eq. (2) can be brought down to less than $4 \%$ in the measurement of the jet nuclear modification factor [19]. In addition, a precise determination of Eq. (2) requires controlling the $\mathrm{OO}$ and $p p$ beam luminosities with comparable accuracy [36,37]. In this case, both the experimental precision and theoretical accuracy of the no-parton-energy-loss baseline of Eq. (2) in $\mathrm{OO}$ would be high enough to provide unprecedented sensitivity for the search of parton energy loss in systems with $\left\langle N_{\text {part }}\right\rangle \sim 10$.

In close analogy, we have also calculated the nuclear modification factor, Eq. (2), for single inclusive charged hadron spectra at LO and NLO. We convoluted the parton spectra with Binnewies-Kniehl-Kramer (BKK) [38] and Kniehl-Kramer-Potter (KKP) [39] fragmentation functions (FFs) using the INCNLO program $[40,41]$ modified to use LHAPDF grids [42]. We obtained hadronic FFs by summing pion and kaon FFs for BKK and pion and kaon and proton FFs for KKP. We checked that BKK FFs (our default choice) provide a reasonable description of the measured charged hadron spectra at $\sqrt{s}=7 \mathrm{TeV} p p$ collisions. In the absence of final state rescattering in the QCD medium, the same FFs enter the numerator and the denominator in Eq. (2), such that the ratio is largely insensitive to the specific choice of FFs, as shown in Fig. 3. The remaining uncertainty is dominated again by our current knowledge of nPDFs. As parton fragmentation softens hadron distributions, the region of small $\sim 2 \%$ uncertainty lies at a $p_{T}$ that is shifted compared to the $p_{T}$ dependence in Fig. 2.

Predictions of parton energy loss. - The sizable azimuthal momentum anisotropies $v_{n}$ observed in systems of $\left\langle N_{\text {part }}\right\rangle \sim 10$ are interpreted in terms of interactions in the QCD medium. Therefore, qualitatively, some parton energy loss in $\mathrm{OO}$ collisions is expected. However, quantitative theoretical expectations for $R_{\mathrm{AA} \text {, min bias }}^{h}$ are model dependent, and there is no a priori reason that the effect is large. The medium modifications of the multiparticle final states

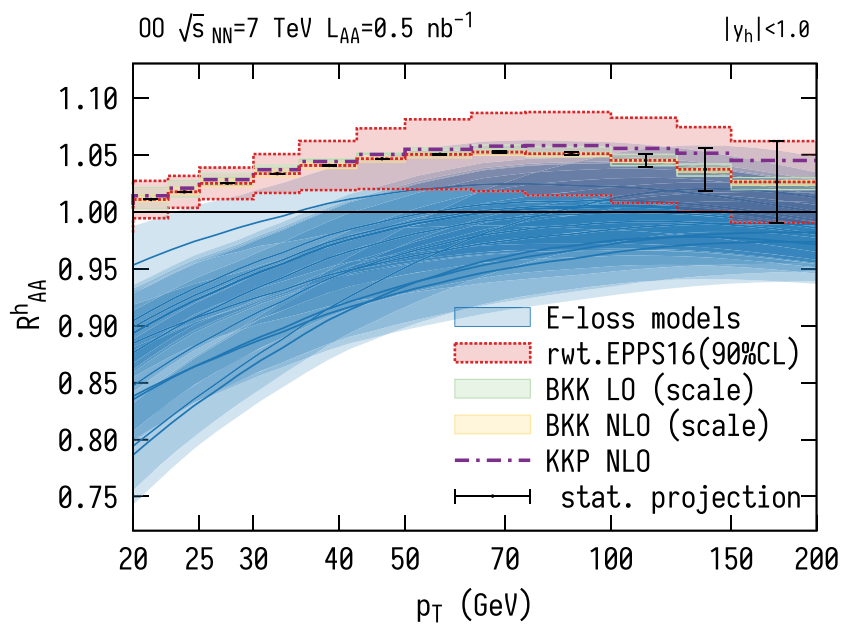

FIG. 3. Minimum bias hadron nuclear modification factor, Eq. (2), for OO collisions. A broad range of parton energy loss model predictions (blue bands) [43] is overlaid with the baseline in the absence of parton rescattering. The red band shows a reweighted nPDF 90\% confidence level (reweighting is done by including additional $p \mathrm{~Pb}$ dijet data). Proton PDF (not shown), scale (green and yellow) uncertainties are fully correlated and cancel. Dot-dashed line shows central NLO prediction with KKP FFs. Error bars illustrate statistical uncertainties for $\mathrm{OO}$ mock data at $100 \%$ efficiency. 
giving rise to jets are more complicated to model than single inclusive hadron spectra, and none of the Monte Carlo tools developed to this end (see, e.g., [44-46]) have been tuned to very small collision systems. For these reasons, we restrict the following discussion of quantitative model expectations for parton energy loss in OO to single inclusive hadron spectra.

In general, models of parton energy loss supplement the framework of collinearly factorized QCD with assumptions about the rescattering and ensuing modifications of the final state parton shower in the QCD medium. For leading hadron spectra, the hard matrix elements are typically convoluted with quenching weights that characterize the parton energy loss of the leading parton in the QCD medium prior to hadronization in the vacuum. First perturbative calculations of this parton rescattering within QCD go back to the works of Baier-Dokshitzer-MuellerPeigne-Schiff and Zakharov [47-50] and many others [51-53]. Within this framework, a large number of models were developed for the description of $R_{\mathrm{AA}}^{h}$ over the last two decades [54]. These models differ in their assumptions about the strength of the rescattering (typically parameterized in terms of the quenching parameter $\hat{q}$ or an equivalent parameter), the time evolution of the medium, the path length dependence, and other details. To the best of our knowledge, none of these models have been used to make predictions for $R_{\mathrm{AA}}^{h}$ min bias in OO collisions.

In a companion paper [43], we therefore derive predictions for $R_{\mathrm{AA} \text {, min bias }}^{h}$ in $\mathrm{OO}$ collisions. This is done by building a simple modular version of the factorized perturbative QCD framework supplemented with parton energy loss. We have systematically tested the resulting $R_{\text {AA,min bias }}^{h}\left(p_{T}\right)$ for a wide set of model assumptions. All models were tuned to experimental data of $R_{\mathrm{AA}, \min \text { bias }}^{h}\left(p_{T}\right)$ in $\sqrt{s_{N N}}=5.02 \mathrm{TeV} \mathrm{PbPb}$ collisions at $p_{T} \sim 50 \mathrm{GeV}$ [17]. We then predict the $p_{T}$ and system size dependence. Although our procedure is not the same as reproducing the various published parton energy loss models (the different model assumptions are embedded all in the same simple setup), we expect that this characterizes reasonably well the spread in model predictions for $\mathrm{OO}$ collisions. Referring for details to the companion paper [43], we show the final result in Fig. 3. The blue lines result from overlaying predictions for different modeling assumptions and thus presents a robust expectation for parton energy loss. The blue bands represent model and (reweighted) nPDF uncertainties added in quadrature. We conclude that a $15 \%$ uncertainty in modeling of $\left\langle T_{\mathrm{AA}}\right\rangle$ in very peripheral $\mathrm{PbPb}$ collisions would prevent separating a large fraction of the model predictions from the null hypothesis. However, the much improved theoretical accuracy of Eq. (2) (error bands in Fig. 3) allows for this separation for the large majority of models in the range of $20 \mathrm{GeV}<p_{T}<50 \mathrm{GeV}$ and for some in the range up to $100 \mathrm{GeV}$.
Opportunities of Z-boson measurements.-While our model studies indicate that the theoretical accuracy will be sufficient to discover partonic rescattering in small systems, the use of Eq. (2) could potentially be limited by beam luminosity uncertainties. $Z$-boson production has been long touted as a golden channel to measure precisely the hard partonic luminosity $[55,56]$. Therefore, we consider the $Z$-boson normalized nuclear modification factor

$$
R_{\mathrm{AA}, Z}^{h, j}\left(p_{T}, y\right)=\frac{\sigma_{p p}^{Z}}{\sigma_{\mathrm{AA}}^{Z}} \frac{d \sigma_{\mathrm{AA}}^{h, j} / d p_{T} d y}{d \sigma_{p p}^{h, j} / d p_{T} d y} .
$$

In comparison to Eq. (2), this measurement has the additional advantage of the beam luminosity uncertainties canceling in the double ratio of cross sections.

OO collisions at LHC can reach an order of magnitude larger effective nucleon-nucleon luminosity than $\mathrm{PbPb}$ collisions [15]. A sample of $\mathcal{O}\left(10^{5}\right) Z$ bosons can be recorded with an integrated luminosity $\mathcal{L}_{A A}=0.5 \mathrm{pb}^{-1}$ of OO collisions, which corresponds nominally to $\mathcal{O}$ (1day) stable running at LHC. This would bring the statistical uncertainties of the normalization in Eq. (3) below $1 \%$.

As both jet and Z-boson yields are proportional to the incoming parton flux, we expected that the nPDF uncertainties would also largely cancel in the double ratio. In Fig. 4 we show the baseline calculation of Eq. (3) obtained in the same NNLOJET framework and displayed with the same breakdown of theoretical uncertainties as Fig. 2. The comparison of Figs. 2 and 4 makes it clear that our initial assumption was wrong and that the nPDF uncertainties in Eq. (3) are larger than those in Eq. (2). The reason for this is that the $Z$-boson and jet cross sections probe different Bjorken- $x$ ranges and that the nPDF uncertainties of these

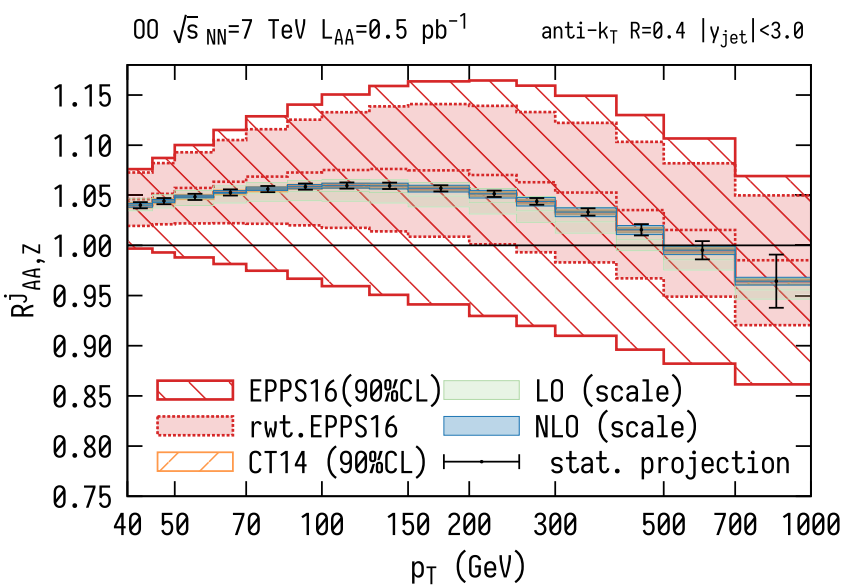

FIG. 4. (a) The Z-boson normalized jet nuclear modification factor, Eq. (3), for $\mathrm{OO}$ collisions in the absence of parton rescattering (analogous to Fig. 2). The surprising increase in the red band is due to the anticorrelation of $Z$ and jet nPDF uncertainties (see text). The error bars represent statistical uncertainties of OO mock data at $100 \%$ efficiency at an integrated luminosity of $\mathcal{L}_{\mathrm{AA}}=0.5 \mathrm{pb}^{-1}$. 
ranges turn out to be anticorrelated (see the Supplemental Material [35]). We conclude that the theoretical accuracy of Z-boson normalized nuclear modification factor, Eq. (3), relies on a precise knowledge of nPDFs. As more LHC data on AA and $p$ A collision will be included in the nPDF fits, $n P D F$ uncertainties will be reduced. It would be interesting to study to what extent future $p \mathrm{O}$ and $\mathrm{OO}$ runs at LHC can improve the current nPDF uncertainties.

Summary.-We have started from the observation that the current characterization of parton energy loss in small systems relies on centrality dependent measurements whose construction depends on assumptions about soft physics (in particular manifest in $\left\langle T_{\mathrm{AA}}\right\rangle$ ). The associated uncertainties are difficult to improve systematically, and they constitute a significant limitation for high precision measurements of small parton energy loss effects in small collision systems. We have demonstrated with LO and NLO calculations of the baseline of negligible parton energy loss that theoretical uncertainties for inclusive measurements of nuclear modification factors are much smaller and as low as $2 \%$ in the kinematically most favorable regions. Moreover, these uncertainties can be systematically improved with new data that constrain nPDFs.

We reemphasize that partonic rescattering is a prerequisite for quark-gluon plasma formation and that partonic rescattering is a direct logical consequence of the standard interpretation of azimuthal anisotropies $v_{n}$ in terms of final state interactions. The possibility that $v_{n}$ is observed while partonic scattering is absent contradicts such phenomenological interpretations of heavy-ion data. The discovery of parton energy loss in small collision systems is therefore one of the most important challenges of the future experimental heavy-ion program. Here, we have shown that the improved theoretical uncertainty in the baseline calculation of inclusive hadron spectra is needed to separate unambiguously model predictions of partonic rescattering from the null hypothesis in the small $\mathrm{OO}$ collision system. The integrated luminosity to make this possible is $\mathcal{O}\left(1 \mathrm{nb}^{-1}\right)$. Measurements of $Z$-boson normalized $R_{\mathrm{AA}, Z}$ would provide an alternative characterization of parton energy loss in OO collisions that has comparable accuracy and that has the advantage of the luminosity uncertainties canceling. Such measurements requires an integrated luminosity of $\mathcal{O}\left(1 \mathrm{pb}^{-1}\right)$. We hope that our proposal helps to clarify one of the main outstanding questions in the LHC heavy-ion program and that it informs the ongoing discussions about the integrated luminosity required to exploit the unique opportunities of an OO run at the LHC.

We thank Rabah Abdul Khalek, Julien Baglio, Valerio Bertone, Roderik Bruce, Silvia Ferrario Ravasio, Oscar Garcia-Montero, Marc Andre Jebramcik, John Jowett, YenJie Lee, Andreas Morsch, Dennis Perepelitsa, Juan Rojo, Marta Verweij, and Eleni Vryonidou for valuable discussions. We thank Hannu Paukkunen for providing EPPS16 parton distribution functions for oxygen, Valerio Bertone for sharing the modified INCNLO code, and Guilherme Milhano for numerous discussions throughout the project.

*Corresponding author. alexander.huss@cern.ch ${ }^{\dagger}$ Corresponding author. a.k@cern.ch Corresponding author. aleksas.mazeliauskas@cern.ch ${ }^{\S}$ Corresponding author. risto.sakari.paatelainen@cern.ch "Corresponding author. wilke.van.der.schee@cern.ch

"Corresponding author. urs.wiedemann@cern.ch

[1] K. Adcox et al. (PHENIX Collaboration), Phys. Rev. Lett. 88, 022301 (2001).

[2] C. Adler et al. (STAR Collaboration), Phys. Rev. Lett. 89, 202301 (2002).

[3] K. Aamodt et al. (ALICE Collaboration), Phys. Lett. B 696, 30 (2011).

[4] S. Chatrchyan et al. (CMS Collaboration), Eur. Phys. J. C 72, 1945 (2012).

[5] G. Aad et al. (ATLAS Collaboration), J. High Energy Phys. 09 (2015) 050.

[6] B. B. Abelev et al. (ALICE Collaboration), Phys. Rev. C 90, 054901 (2014).

[7] M. Aaboud et al. (ATLAS Collaboration), Eur. Phys. J. C 77, 428 (2017).

[8] B. B. Abelev et al. (ALICE Collaboration), Phys. Lett. B 728, 216 (2014); 734, 409(E) (2014).

[9] B. B. Abelev et al. (ALICE Collaboration), Phys. Rev. Lett. 111, 222301 (2013).

[10] J. Adam et al. (ALICE Collaboration), Phys. Lett. B 754, 235 (2016).

[11] J. Adam et al. (ALICE Collaboration), Phys. Lett. B 758, 389 (2016).

[12] J. Adam et al. (ALICE Collaboration), Nat. Phys. 13, 535 (2017).

[13] V. Khachatryan et al. (CMS Collaboration), Phys. Rev. Lett. 115, 012301 (2015).

[14] V. Khachatryan et al. (CMS Collaboration), Phys. Lett. B 765, 193 (2017).

[15] Z. Citron et al., in Report on the Physics at the HL-LHC, and Perspectives for the HE-LHC, edited by A. Dainese, M. Mangano, A. B. Meyer, A. Nisati, G. Salam, and M. A. Vesterinen, CERN Yellow Rep. Monogr. (CERN, Geneva, 2019), 1159, https://doi.org/10.23731/CYRM-2019-007 .1159 .

[16] J. Adolfsson et al., Eur. Phys. J. A 56, 288 (2020).

[17] V. Khachatryan et al. (CMS Collaboration), J. High Energy Phys. 04 (2017) 039.

[18] A. M. Sirunyan et al. (CMS Collaboration), J. High Energy Phys. 10 (2018) 138.

[19] M. Aaboud et al. (ATLAS Collaboration), Phys. Lett. B 790, 108 (2019).

[20] G. Aad et al. (ATLAS Collaboration), Phys. Lett. B 748, 392 (2015). 
[21] R. Glauber and G. Matthiae, Nucl. Phys. B21, 135 (1970).

[22] D. G. d'Enterria, arXiv:nucl-ex/0302016.

[23] M. L. Miller, K. Reygers, S. J. Sanders, and P. Steinberg, Annu. Rev. Nucl. Part. Sci. 57, 205 (2007).

[24] C. Loizides and A. Morsch, Phys. Lett. B 773, 408 (2017).

[25] K. J. Eskola, I. Helenius, M. Kuha, and H. Paukkunen, Phys. Rev. Lett. 125, 212301 (2020).

[26] J. Currie, E. W. N. Glover, and J. Pires, Phys. Rev. Lett. 118, 072002 (2017).

[27] T. Gehrmann et al., Proc. Sci., RADCOR2017 (2018) 074 [arXiv:1801.06415].

[28] D. Britzger et al., Eur. Phys. J. C 79, 845 (2019).

[29] S. Dulat, T.-J. Hou, J. Gao, M. Guzzi, J. Huston, P. Nadolsky, J. Pumplin, C. Schmidt, D. Stump, and C. P. Yuan, Phys. Rev. D 93, 033006 (2016).

[30] S. Alioli, K. Hamilton, P. Nason, C. Oleari, and E. Re, J. High Energy Phys. 04 (2011) 081.

[31] K. J. Eskola, P. Paakkinen, H. Paukkunen, and C. A. Salgado, Eur. Phys. J. C 77, 163 (2017).

[32] A. M. Sirunyan et al. (CMS Collaboration), Phys. Rev. Lett. 121, 062002 (2018).

[33] H. Paukkunen and P. Zurita, J. High Energy Phys. 12 (2014) 100.

[34] K. J. Eskola, P. Paakkinen, and H. Paukkunen, Eur. Phys. J. C 79, 511 (2019).

[35] See Supplemental Material at http://link.aps.org/ supplemental/10.1103/PhysRevLett.126.192301 for the summary of the nPDF reweighting procedure and the analysis of Z-boson and jet cross section correlations.

[36] ATLAS collaboration, Technical Report No. ATLASCONF-2019-021, CERN, Geneva, 2019, https://cds.cern .ch/record/2677054.

[37] ATLAS collaboration, Technical Report No. ATLASCONF-2020-010, CERN, Geneva, 2020, http://cdsweb .cern.ch/record/2719516/.
[38] J. Binnewies, B. A. Kniehl, and G. Kramer, Z. Phys. C 65, 471 (1995).

[39] B. A. Kniehl, G. Kramer, and B. Potter, Nucl. Phys. B582, 514 (2000).

[40] F. Aversa, P. Chiappetta, M. Greco, and J. Guillet, Nucl. Phys. B327, 105 (1989).

[41] http://lapth.cnrs.fr/PHOX_FAMILY/readme_inc.html.

[42] A. Buckley, J. Ferrando, S. Lloyd, K. Nordstrm, B. Page, M. Rfenacht, M. Schnherr, and G. Watt, Eur. Phys. J. C 75, 132 (2015).

[43] A. Huss, A. Kurkela, A. Mazeliauskas, R. Paatelainen, W. van der Schee, and U. A. Wiedemann, companion paper, Phys. Rev. C 103, 054903 (2021).

[44] K. C. Zapp, Eur. Phys. J. C 74, 2762 (2014).

[45] J. Putschke et al., arXiv:1903.07706.

[46] B. Schenke, C. Gale, and S. Jeon, Phys. Rev. C 80, 054913 (2009).

[47] R. Baier, Y. L. Dokshitzer, A. H. Mueller, S. Peigne, and D. Schiff, Nucl. Phys. B483, 291 (1997).

[48] R. Baier, Y. L. Dokshitzer, A. H. Mueller, S. Peigne, and D. Schiff, Nucl. Phys. B484, 265 (1997).

[49] B. Zakharov, JETP Lett. 63, 952 (1996).

[50] B. Zakharov, JETP Lett. 65, 615 (1997).

[51] U. A. Wiedemann, Nucl. Phys. B588, 303 (2000).

[52] M. Gyulassy, P. Levai, and I. Vitev, Nucl. Phys. B594, 371 (2001).

[53] X.-N. Wang and X.-F. Guo, Nucl. Phys. A696, 788 (2001).

[54] N. Armesto et al., Phys. Rev. C 86, 064904 (2012).

[55] M. Dittmar, F. Pauss, and D. Zurcher, Phys. Rev. D 56, 7284 (1997).

[56] V. A. Khoze, A. D. Martin, R. Orava, and M. Ryskin, Eur. Phys. J. C 19, 313 (2001). 